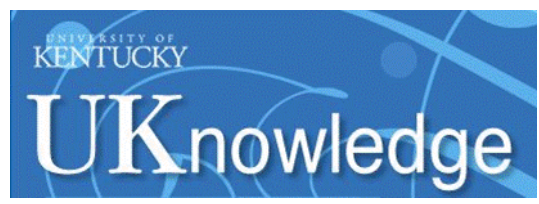

University of Kentucky

UKnowledge

$10-2014$

\title{
Crystal Structure of
}

\section{(E)-13-\{4-[(Z)-2-cyano-2-(3,4,5-trimethoxyphenyl)ethenyl]phenyl\}parthenolide methanol hemisolvate}

\author{
Narsimha Reddy Penthala \\ University of Arkansas \\ Shobanbabu Bommagani \\ University of Arkansas \\ Venumadhav Janganati \\ University of Arkansas \\ Sean Parkin \\ University of Kentucky, s.parkin@uky.edu \\ Peter A. Crooks \\ University of Arkansas, pacrooks@uams.edu
}

Follow this and additional works at: https://uknowledge.uky.edu/chemistry_facpub

Part of the Chemistry Commons

Right click to open a feedback form in a new tab to let us know how this document benefits you.

\section{Repository Citation}

Penthala, Narsimha Reddy; Bommagani, Shobanbabu; Janganati, Venumadhav; Parkin, Sean; and Crooks, Peter A., "Crystal Structure of (E)-13-\{4-[(Z)-2-cyano-2-(3,4,5-trimethoxyphenyl)ethenyl]phenyl\}parthenolide methanol hemisolvate" (2014). Chemistry Faculty Publications. 30.

https://uknowledge.uky.edu/chemistry_facpub/30

This Article is brought to you for free and open access by the Chemistry at UKnowledge. It has been accepted for inclusion in Chemistry Faculty Publications by an authorized administrator of UKnowledge. For more information, please contact UKnowledge@lsv.uky.edu. 


\section{Crystal Structure of}

(E)-13-\{4-[(Z)-2-cyano-2-(3,4,5-trimethoxyphenyl)ethenyl]phenyl\}parthenolide methanol hemisolvate

Digital Object Identifier (DOI)

https://doi.org/10.1107/S1600536814019333

Notes/Citation Information

Published in Acta Crystallographica Section E: Crystallographic Communications, v. 70, part 10, p. o1092-01093.

This is an open-access article distributed under the terms of the Creative Commons Attribution License, which permits unrestricted use, distribution, and reproduction in any medium, provided the original authors and source are cited. 


\section{STRUCTURE REPORTS}

OPEN $\curvearrowright$ ACCESS

ISSN 1600-5368

Crystal structure of $(E)-13-\{4-[(Z)-2-$ cyano-2-(3,4,5-trimethoxyphenyl)ethenyl]phenyl\}parthenolide methanol hemisolvate

\begin{abstract}
Narsimha Reddy Penthala, ${ }^{a}$ Shobanbabu Bommagani, Venumadhav Janganati, ${ }^{a}$ Sean Parkin ${ }^{b}$ and Peter A. Crooks $^{\mathrm{a} *}$

${ }^{\mathbf{a}}$ Department of Pharmaceutical Sciences, College of Pharmacy, University of Arkansas for Medical Sciences, Little Rock, AR 72205, USA, and ${ }^{\mathbf{b}}$ Department of Chemistry, University of Kentucky, Lexington KY 40506, USA. *Correspondence e-mail: pacrooks@uams.edu
\end{abstract}

Received 22 May 2014; accepted 26 August 2014

Edited by J. Simpson, University of Otago, New Zealand

The title compound, $\mathrm{C}_{33} \mathrm{H}_{35} \mathrm{NO}_{6}$ [systematic name: $(Z)-3-(4-$ $\{(E)$-[(E)-1a,5-dimethyl-9-oxo-2,3,7,7a-tetrahydrooxireno$\left[2^{\prime}, 3^{\prime}: 9,10\right]$ cyclodeca[1,2-b]furan-8(1a $\left.H, 6 H, 9 H, 10 \mathrm{a} H, 10 \mathrm{~b} H\right)$-ylidene]methyl\}phenyl)-2-(3,4,5-trimethoxyphenyl)acrylonitrile methanol hemisolvate], $\mathrm{C}_{33} \mathrm{H}_{35} \mathrm{NO}_{6} \cdot 0.5 \mathrm{CH}_{3} \mathrm{OH}$, was prepared by the reaction of (Z)-3-(4-iodophenyl)-2-(3,4,5-trimethoxyphenyl)acrylonitrile with parthenolide [systematic name: (E)-1a,5-dimethyl-8-methylene-2,3,6,7,7a,8,10a,10b-octahydrooxireno[ $\left[2^{\prime}, 3^{\prime}: 9,10\right]$ cyclodeca[1,2- $\left.b\right]$ furan- $9(1 \mathrm{a} H)$-one $]$ under Heck reaction conditions. The molecule is built up from fused ten-, five- (lactone) and three-membered (epoxide) rings with a $\{4-[(Z)-2$-cyano-2-(3,4,5-trimethoxyphenyl)ethenyl]phenyl $\}$ methylidene group as a substituent. The $4-[(Z)-2-$ cyano-2-(3,4,5-trimethoxyphenyl)ethenyl]phenyl group on the parthenolide exocyclic double bond is oriented in a trans position to the lactone ring to form the $E$ isomer. The dihedral angle between the benzene ring of the phenyl moiety and the lactone ring mean plane is $21.93(4)^{\circ}$.

Keywords: crystal structure; parthenolide derivatives; Heck synthesis; biological activity.

CCDC reference: 1021449

\section{Related literature}

For the biological activity of parthenolide, see: Hall et al. (1979). For the biological activity of parthenolide derivatives similar to the title compound, see: Hanson et al. (1970); Hehner et al. (1998); Kupchan et al. (1971); Neelakantan et al.
(2009); Oka et al., 2007); Ralstin et al. (2006); Sun et al. (2006); Penthala et al. (2013b). For the synthesis and crystal structures of similar molecules, see: Han et al. (2009); Penthala et al. (2013a). For details of the experimental procedure, see: Hope (1994); Parkin \& Hope (1998);

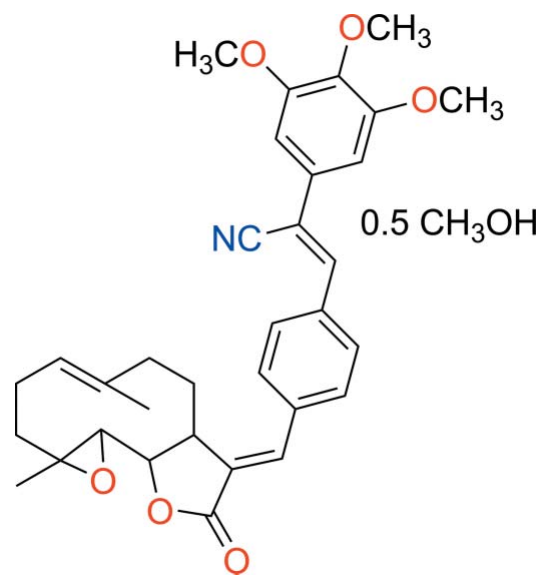

\section{Experimental}

\subsection{Crystal data}

$\mathrm{C}_{33} \mathrm{H}_{35} \mathrm{NO}_{6} \cdot 0.5 \mathrm{CH}_{4} \mathrm{O}$

$M_{r}=557.64$

Orthorhombic, $P 2_{1} 2_{1} 2_{1}$

$a=9.3347(2) \AA$

$b=16.2442(3) \AA$

$c=19.2580$ (4) $\AA$

$$
\begin{aligned}
& V=2920.18(10) \AA^{3} \\
& Z=4 \\
& \text { Cu K } \alpha \text { radiation } \\
& \mu=0.71 \mathrm{~mm}^{-1} \\
& T=90 \mathrm{~K} \\
& 0.18 \times 0.15 \times 0.10 \mathrm{~mm}
\end{aligned}
$$

\subsection{Data collection}

Bruker X8 Proteum diffractometer Absorption correction: multi-scan (SADABS; Sheldrick, 2008b)

$T_{\min }=0.836, T_{\max }=0.963$

40379 measured reflections 5349 independent reflections 5303 reflections with $I>2 \sigma(I)$ $R_{\text {int }}=0.036$

\subsection{Refinement}

$R\left[F^{2}>2 \sigma\left(F^{2}\right)\right]=0.024$

$w R\left(F^{2}\right)=0.065$

$S=1.03$

5349 reflections

387 parameters

$\mathrm{H}$-atom parameters constrained

$\Delta \rho_{\max }=0.14{\mathrm{e} \AA^{-3}}^{-3}$

Data collection: $A P E X 2$ (Bruker, 2006); cell refinement: $A P E X 2$; data reduction: $A P E X 2$; program(s) used to solve structure: SHELXS97 (Sheldrick, 2008a); program(s) used to refine structure: SHELXL2014 (Sheldrick, 2008a); molecular graphics: $X P$ in SHELXTL (Sheldrick, 2008a); software used to prepare material for publication: SHELXL97 (Sheldrick, 2008a), CIFFIX (Parkin, 2013), PLATON (Spek, 2009) and local program (Parkin, 2000).

\section{Acknowledgements}

This work was supported by NIH/NCI (grant No. CA158275). 
Supporting information for this paper is available from the IUCr electronic archives (Reference: SJ5404).

\section{References}

Bruker (2006). APEX2. Bruker AXS Inc., Madison, Wisconsin, USA.

Hall, I. H., Lee, K. H., Starnes, C. O., Sumida, Y., Wu, R. Y., Waddell, T. G., Cochran, J. W. \& Gerhart, K. G. (1979). J. Pharm. Sci. 68, 537-542.

Han, C., Barrios, F. J., Riofski, M. V. \& Colby, D. A. (2009). J. Org. Chem. 74, 7176-7179.

Hanson, R. L., Lardy, H. A. \& Kupchan, S. M. (1970). Science, 168, 378-380.

Hehner, S. P., Heinrich, M., Bork, P. M., Vogt, M., Ratter, F., Lehmann, V., Osthoff, K. S., Dröge, W. \& Schmitz, M. L. (1998). J. Biol. Chem. 273, 12881297.

Hope, H. (1994). Prog. Inorg. Chem. 41, 1-19.

Kupchan, S. M., Eakin, M. A. \& Thomas, A. M. (1971). J. Med. Chem. 14, $1147-1152$.
Neelakantan, S., Nasim, S., Guzman, M. L., Jordan, C. T. \& Crooks, P. A. (2009). Bioorg. Med. Chem. Lett. 19, 4346-4349.

Oka, D., Nishimura, K., Shiba, M., Nakai, Y., Arai, Y., Nakayama, M., Takayama, H., Inoue, H., Okuyama, A. \& Nonomura, N. (2007). Int. J. Cancer, 120, 2576-2581.

Parkin, S. (2000). Acta Cryst. A56, 157-162.

Parkin, S. (2013). CIFFIX, http://xray.uky.edu/people/parkin/programs/ciffix. Parkin, S. \& Hope, H. (1998). J. Appl. Cryst. 31, 945-953.

Parsons, S., Flack, H. D. \& Wagner, T. (2013). Acta Cryst. B69, 249-259.

Penthala, N. R., Janganati, V., Parkin, S., Varughese, K. I. \& Crooks, P. A. (2013a). Acta Cryst. E69, o1709-o1710.

Penthala, N. R., Sonar, V. N., Horn, J., Leggas, M., Yadlapalli, J. S. \& Crooks, P. A. (2013b). Medchemcomm, 4, 1073-1078.

Ralstin, M. C., Gage, E. A., Yip-Schneider, M. T., Klein, P. J., Wiebke, E. A. \& Schmidt, C. M. (2006). Mol. Cancer Res. 4, 387-399.

Sheldrick, G. M. (2008a). Acta Cryst. A64, 112-122.

Sheldrick, G. M. (2008b). SADABS. University of Göttingen, Germany.

Spek, A. L. (2009). Acta Cryst. D65, 148-155.

Sun, H.-X., Zheng, Q.-F. \& Tu, J. (2006). Bioorg. Med. Chem. 14, 1189-1198. 


\title{
supporting information
}

Acta Cryst. (2014). E70, o1092-o1093［doi:10.1107/S1600536814019333]

\section{Crystal structure of $(E)-13-\{4-[(Z)-2-c y a n o-2-(3,4,5-$ trimethoxyphenyl)- ethenyl]phenyl\}parthenolide methanol hemisolvate}

\author{
Narsimha Reddy Penthala, Shobanbabu Bommagani, Venumadhav Janganati, Sean Parkin and \\ Peter A. Crooks
}

\section{S1. Comment}

Parthenolide (PTL) and its analogs belong to the class of sesquiterpene lactones. These compounds are currently being used in the development of anti-cancer agents for the treatment of hematological tumours (Sun et al., 2006; Hehner et al., 1998; Ralstin et al. 2006; Oka et al., 2007; Kupchan et al., 1971 and Hanson et al., 1970). Recently, we have reported the crystal structure of (E)-13-(4-aminophenyl)parthenolide, a Heck reaction derivative of parthenolide (Penthala et al. 2013a), and we have also reported on Z-2-(3,4,5-trimethoxyphenyl)acrylonitrile analogs (Penthala et al. 2013b) as anticancer agents. As part of a program for the development of parthenolide analogs as anti-leukemic agents (Neelakantan $e t$ al. 2009), and small molecule analogs as anti-cancer agents, our research group is focusing on the synthesis of $E$-olefinic analogues of PTL which can be obtained from the reaction of parthenolide with iodoaromatic reagents utilizing Heck chemistry (Han et al. 2009). The title compound was obtained from the reaction of parthenolide with (Z)-3-(4-iodophenyl) -2-(3,4,5- trimethoxyphenylacrylonitrile under Heck reaction conditions. To obtain detailed information on the structure of the title compound and to establish the geometry of the exocyclic $\mathrm{C} 13-\mathrm{C} 14$ double bond, a single-crystal Xray structure determination has been carried out.

Recrystallization of the title compound from methanol afforded light yellow coloured crystals that were suitable for Xray analysis. The X-ray studies revealed that the title compound was identified as the $E$-isomer (conformation about the exocyclic methylidene $\mathrm{C}=\mathrm{C}$ bond; the conformation about the $\mathrm{C}=\mathrm{C}$ bond in the ten-membered ring is also $E$ ). The molecule is built up from fused ten-, five- (lactone) and three-membered (epoxide) rings with a (Z)-3-(4-phenyl)-2-(3,4,5trimethoxyphenyl)acrylonitrile group as a substituent. The dihedral angle between the benzene ring of the phenyl moiety and the lactone ring mean plane is 21.93 (4) $\AA$.

\section{S2. Experimental}

A mixture of parthenolide (1.0 mmol), diisopropylethylamine (3.0 mmol), and (Z)-3-(4-iodophenyl)-2-(3,4,5-trimethoxyphenyl acrylonitrile $(1.1 \mathrm{mmol})$ in toluene $(1 \mathrm{ml})$ was treated with palladium (II) ferrocene $(0.01 \mathrm{mmol})$ and then stirred at $353 \mathrm{~K}$ for $24 \mathrm{~h}$. The reaction mixture was cooled to room temperature, water $(8 \mathrm{ml})$ was added, and the mixture was extracted with ethyl acetate $(10 \mathrm{mlx} 3)$. The separated organics were dried over anhydrous $\mathrm{Na}_{2} \mathrm{SO}_{4}$ and concentrated under reduced pressure. The obtained crude residue was purified using silica flash chromatography (7:3, hexanes/EtOAc) to afford the title compound, which was recrystallized from methanol as light yellow coloured crystals suitable for X-ray analysis (87\% yield; M·P.: 478-480 K); ${ }^{1} \mathrm{H}$ NMR $\left(400 \mathrm{MHz}, \mathrm{CDCl}_{3}\right): \delta 7.95(\mathrm{~d}, J=8.4 \mathrm{~Hz}, 2 \mathrm{H}), 7.67(\mathrm{~d}, J=3.6 \mathrm{~Hz}, 1 \mathrm{H})$, $7.52(\mathrm{~d}, J=8.4 \mathrm{~Hz}, 2 \mathrm{H}), 7.46(\mathrm{~s}, 1 \mathrm{H}), 6.88(\mathrm{~s}, 2 \mathrm{H}), 5.29(\mathrm{~d}, J=11.2 \mathrm{~Hz}, 1 \mathrm{H}), 3.94\left(\mathrm{~s}, 6 \mathrm{H}, 2 \mathrm{xOCH}_{3}\right), 3.90\left(\mathrm{~s}, 3 \mathrm{H}, \mathrm{OCH}_{3}\right)$, $3.3(\mathrm{~m}, 1 \mathrm{H}), 2.85(\mathrm{~d}, J=8.4 \mathrm{~Hz}, 1 \mathrm{H}), 2.41-2.46(\mathrm{~m}, 1 \mathrm{H}), 2.10-2.27(\mathrm{~m}, 5 \mathrm{H}), 1.69\left(\mathrm{~s}, 3 \mathrm{H}, \mathrm{CH}_{3}\right), 1.46-1.55(\mathrm{~m}, 2 \mathrm{H}), 1.32$ 


\section{supporting information}

(s, 3H, $\left.\mathrm{CH}_{3}\right), 1.27-1.30(\mathrm{~m}, 1 \mathrm{H})$ p.p.m. ${ }^{13} \mathrm{C} \mathrm{NMR}\left(100 \mathrm{MHz}, \mathrm{CDCl}_{3}\right): \delta 17.60,17.70,24.54,30.58,36.33,42.13,47.16$, 55.60, 61.27, 61.94, 66.71, 83.30, 103.75, 113.21, 118.04, 125.48, 129.53, 129.95, 130.56, 130.97, 134.88, 134.96, $135.69,137.06,139.77,140.34,153.89,170.85$ p.p.m. .

\section{S3. Refinement}

Crystal data, data collection and structure refinement details are summarized in Table 1. $\mathrm{H}$ atoms were found in difference Fourier maps, but subsequently included in the refinement using riding models, with constrained distances set to $0.95 \% \mathrm{~A}\left(\mathrm{C}_{\mathrm{sp} 2} \mathrm{H}\right), 0.98 \AA\left(\mathrm{RCH}_{3}\right), 0.99 \AA\left(R_{2} \mathrm{CH}_{2}\right), 1.00 \AA\left(R_{3} \mathrm{CH}\right)$ and $0.84 \AA(\mathrm{OH}) . U_{\text {iso }}(\mathrm{H})$ parameters were set to values of either $1.2 U_{\text {eq }}$ or $1.5 U_{\text {eq }}\left(\mathrm{RCH}_{3}\right.$ and $\mathrm{OH}$ only) of the attached atom.

The partial occupancy methanol molecule refined to an occupancy of about one half. For the final rounds of refinement its occupancy was fixed at exactly 0.5 for the sake of simplicity. This is reasonable because other crystals from the same batch would almost certainly have had varying amounts of solvent incorporated, due to unpredictable rates of solvent loss dependent on such things as crystal handling. The position of this half-occupancy methanol is consistent with an O$\mathrm{H} \cdots \pi$ weak hydrogen bonding interaction in which the distance between atom O1M and the centroid of the trimethoxyphenyl ring (C24-C29) is $3.212(3) \AA$. 


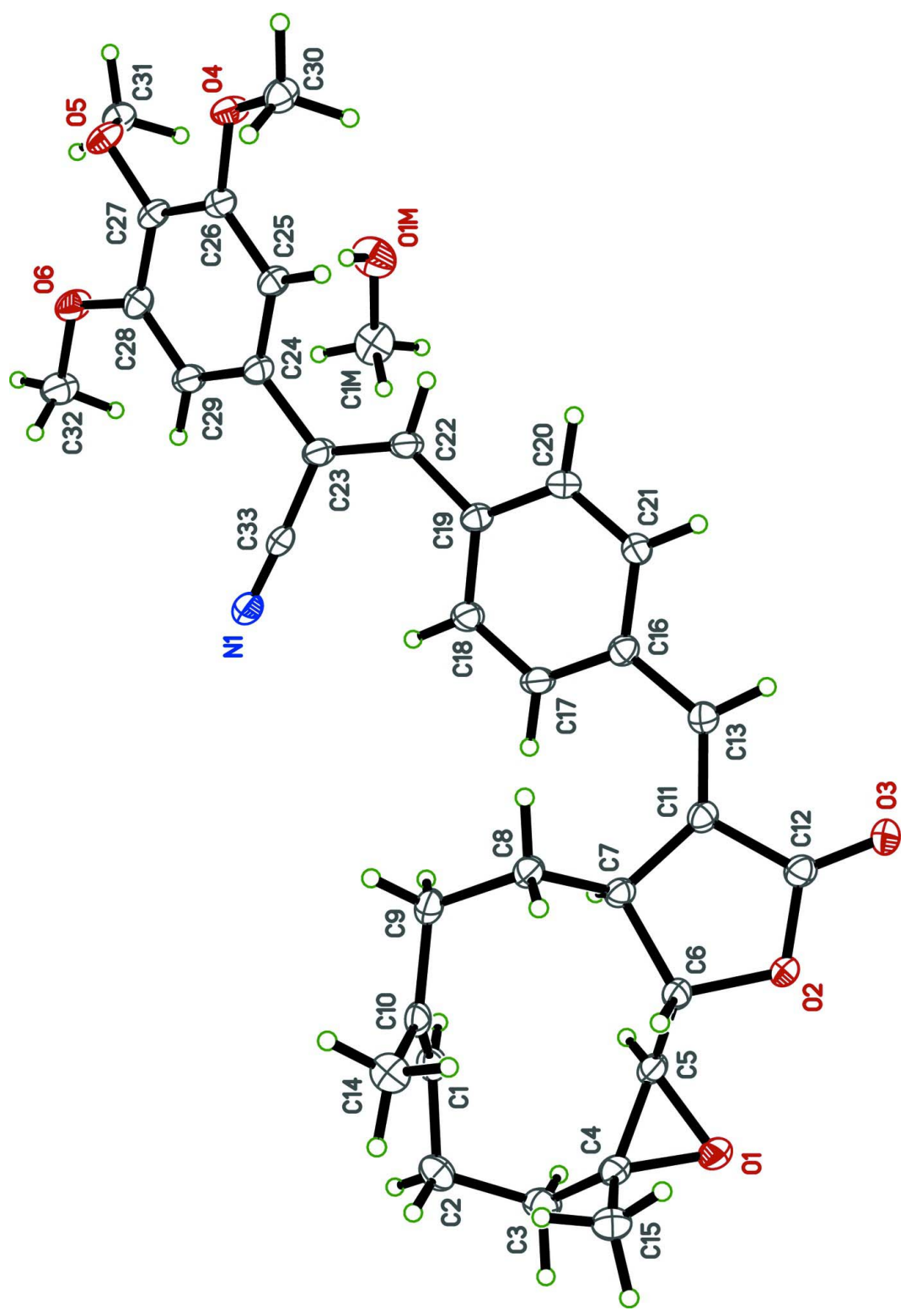

Figure 1

A view of the molecule with displacement ellipsoids drawn at the $50 \%$ probability level.

(Z)-3-(4- $\left\{(E)-\left[(E)-1\right.\right.$ a,5-Dimethyl-9-oxo-2,3,7,7a-tetrahydrooxireno[2', $\left.3^{\prime}: 9,10\right]$ cyclodeca[1,2-

b] furan-8(1aH,6H,9H,10a $H, 10 \mathrm{~b} H)$-ylidene]methyl\}phenyl)-2-(3,4,5-trimethoxyphenyl)acrylonitrile methanol hemisolvate

Crystal data

$\mathrm{C}_{33} \mathrm{H}_{35} \mathrm{NO}_{6} \cdot 0.5 \mathrm{CH}_{4} \mathrm{O}$

$M_{r}=557.64$

Orthorhombic, $P 2{ }_{1} 22_{1}$

$Z=4$

$a=9.3347(2) \AA$

$F(000)=1188$

$b=16.2442(3) \AA$

$D_{\mathrm{x}}=1.268 \mathrm{Mg} \mathrm{m}^{-3}$

$c=19.2580(4) \AA$

$\mathrm{Cu} K \alpha$ radiation, $\lambda=1.54178 \AA$

Cell parameters from 9693 reflections

$V=2920.18(10) \AA^{3}$

$\theta=3.6-68.4^{\circ}$

$\mu=0.71 \mathrm{~mm}^{-1}$ 
$T=90 \mathrm{~K}$

Irregular cut wedge, pale yellow

\section{Data collection}

Bruker X8 Proteum diffractometer

Radiation source: fine-focus rotating anode

Detector resolution: 5.6 pixels $\mathrm{mm}^{-1}$

$\varphi$ and $\omega$ scans

Absorption correction: multi-scan

(SADABS; Sheldrick, 2008b)

$T_{\min }=0.836, T_{\max }=0.963$

Refinement

Refinement on $F^{2}$

Least-squares matrix: full

$R\left[F^{2}>2 \sigma\left(F^{2}\right)\right]=0.024$

$w R\left(F^{2}\right)=0.065$

$S=1.03$

5349 reflections

387 parameters

0 restraints

Primary atom site location: structure-invariant direct methods

Secondary atom site location: difference Fourier map

Hydrogen site location: difference Fourier map
$0.18 \times 0.15 \times 0.10 \mathrm{~mm}$

40379 measured reflections

5349 independent reflections

5303 reflections with $I>2 \sigma(I)$

$R_{\text {int }}=0.036$

$\theta_{\max }=68.4^{\circ}, \theta_{\min }=3.6^{\circ}$

$h=-11 \rightarrow 11$

$k=-13 \rightarrow 19$

$l=-20 \rightarrow 23$

$\mathrm{H}$-atom parameters constrained

$w=1 /\left[\sigma^{2}\left(F_{\mathrm{o}}{ }^{2}\right)+(0.0363 P)^{2}+0.5907 P\right]$

where $P=\left(F_{\mathrm{o}}^{2}+2 F_{\mathrm{c}}^{2}\right) / 3$

$(\Delta / \sigma)_{\max }<0.001$

$\Delta \rho_{\max }=0.14$ e $\AA^{-3}$

$\Delta \rho_{\min }=-0.13$ e $\AA^{-3}$

Extinction correction: SHELXL2014 (Sheldrick, 2008a), $\mathrm{Fc}^{*}=\mathrm{kFc}\left[1+0.001 \times \mathrm{xF}^{2} \lambda^{3} / \sin (2 \theta)\right]^{-1 / 4}$

Extinction coefficient: 0.00092 (14)

Absolute structure: Flack $x$ determined using 2283 quotients [(I+)-(I-)]/[(I+)+(I-)] (Parsons et al., 2013)

Absolute structure parameter: $0.02(2)$

Special details

Experimental. The crystal was mounted with polyisobutene oil on the tip of a fine glass fibre, which was fastened in a copper mounting pin with electrical solder. It was placed directly into the cold gas stream of a liquid nitrogen based cryostat, according to published methods (Hope, 1994; Parkin \& Hope, 1998).

Diffraction data were collected with the crystal at $90 \mathrm{~K}$, which is standard practice in this laboratory for the majority of flash-cooled crystals.

Geometry. All e.s.d.'s (except the e.s.d. in the dihedral angle between two 1.s. planes) are estimated using the full covariance matrix. The cell e.s.d.'s are taken into account individually in the estimation of e.s.d.'s in distances, angles and torsion angles; correlations between e.s.d.'s in cell parameters are only used when they are defined by crystal symmetry. An approximate (isotropic) treatment of cell e.s.d.'s is used for estimating e.s.d.'s involving 1.s. planes.

Refinement. Refinement progress was checked using PLATON (Spek, 2009) and by an $R$-tensor (Parkin, 2000). The final model was further checked with the IUCr utility checkCIF.

Fractional atomic coordinates and isotropic or equivalent isotropic displacement parameters $\left(\AA^{2}\right)$

\begin{tabular}{llllll}
\hline & $x$ & $y$ & $z$ & $U_{\text {iso }} * U_{\text {eq }}$ & Occ. $(<1)$ \\
\hline O1 & $0.48351(13)$ & $0.18048(7)$ & $0.54248(6)$ & $0.0242(3)$ & \\
O2 & $0.35142(13)$ & $0.32648(7)$ & $0.48438(5)$ & $0.0210(2)$ & \\
O3 & $0.34451(13)$ & $0.45934(7)$ & $0.45520(6)$ & $0.0237(3)$ & \\
O4 & $0.02191(12)$ & $0.41510(7)$ & $-0.22367(6)$ & $0.0225(3)$ & \\
O5 & $0.14060(12)$ & $0.30869(7)$ & $-0.31036(5)$ & $0.0219(2)$ & \\
O6 & $0.30389(14)$ & $0.18270(7)$ & $-0.26366(6)$ & $0.0261(3)$ & \\
N1 & $0.35753(19)$ & $0.14312(9)$ & $0.01607(8)$ & $0.0311(4)$ & \\
C1 & $0.35744(17)$ & $0.04178(10)$ & $0.37932(8)$ & $0.0203(3)$ & \\
H1 & 0.4318 & 0.0590 & 0.3489 & $0.024^{*}$
\end{tabular}




\begin{tabular}{|c|c|c|c|c|}
\hline $\mathrm{C} 2$ & $0.40090(19)$ & $-0.01340(10)$ & $0.43865(9)$ & $0.0231(3)$ \\
\hline $\mathrm{H} 2 \mathrm{~A}$ & 0.4617 & -0.0586 & 0.4208 & $0.028 *$ \\
\hline $\mathrm{H} 2 \mathrm{~B}$ & 0.3143 & -0.0381 & 0.4598 & $0.028 *$ \\
\hline $\mathrm{C} 3$ & $0.48435(19)$ & $0.03537(10)$ & $0.49458(9)$ & $0.0249(4)$ \\
\hline $\mathrm{H} 3 \mathrm{~A}$ & 0.5000 & 0.0001 & 0.5358 & $0.030 *$ \\
\hline H3B & 0.5792 & 0.0516 & 0.4761 & $0.030^{*}$ \\
\hline $\mathrm{C} 4$ & $0.40199(18)$ & $0.11124(10)$ & $0.51545(8)$ & $0.0213(3)$ \\
\hline $\mathrm{C} 5$ & $0.42653(17)$ & $0.18504(10)$ & $0.47296(8)$ & $0.0187(3)$ \\
\hline H5 & 0.5005 & 0.1772 & 0.4360 & $0.022 *$ \\
\hline C6 & $0.31309(17)$ & $0.24713(9)$ & $0.45417(8)$ & $0.0180(3)$ \\
\hline H6 & 0.2182 & 0.2288 & 0.4727 & $0.022 *$ \\
\hline $\mathrm{C} 7$ & $0.30239(17)$ & $0.26039(9)$ & $0.37424(8)$ & $0.0170(3)$ \\
\hline H7 & 0.3909 & 0.2376 & 0.3522 & $0.020^{*}$ \\
\hline $\mathrm{C} 8$ & $0.17085(17)$ & $0.21982(10)$ & $0.33974(8)$ & $0.0197(3)$ \\
\hline H8A & 0.0932 & 0.2152 & 0.3745 & $0.024 *$ \\
\hline H8B & 0.1362 & 0.2559 & 0.3019 & $0.024^{*}$ \\
\hline C9 & $0.20236(18)$ & $0.13380(10)$ & $0.30982(8)$ & $0.0211(3)$ \\
\hline H9A & 0.1211 & 0.1168 & 0.2801 & $0.025^{*}$ \\
\hline H9B & 0.2887 & 0.1370 & 0.2801 & $0.025^{*}$ \\
\hline $\mathrm{C} 10$ & $0.22602(17)$ & $0.06913(9)$ & $0.36481(8)$ & $0.0189(3)$ \\
\hline $\mathrm{C} 11$ & 0.30617 (16) & $0.35309(9)$ & $0.36819(8)$ & $0.0175(3)$ \\
\hline $\mathrm{C} 12$ & 0.33264 (17) & $0.38809(9)$ & $0.43821(8)$ & $0.0191(3)$ \\
\hline $\mathrm{C} 13$ & $0.29827(17)$ & $0.40414(9)$ & $0.31380(8)$ & $0.0188(3)$ \\
\hline H13 & 0.2959 & 0.4610 & 0.3254 & $0.023 *$ \\
\hline $\mathrm{C} 14$ & $0.09048(18)$ & $0.04115(11)$ & $0.39995(9)$ & 0.0253 (4) \\
\hline $\mathrm{H} 14 \mathrm{~A}$ & 0.1121 & -0.0048 & 0.4313 & $0.038^{*}$ \\
\hline H14B & 0.0213 & 0.0231 & 0.3648 & $0.038^{*}$ \\
\hline $\mathrm{H} 14 \mathrm{C}$ & 0.0498 & 0.0869 & 0.4267 & $0.038^{*}$ \\
\hline $\mathrm{C} 15$ & $0.2653(2)$ & $0.09726(11)$ & $0.55515(9)$ & $0.0265(4)$ \\
\hline $\mathrm{H} 15 \mathrm{~A}$ & 0.2874 & 0.0715 & 0.5999 & $0.040 *$ \\
\hline H15B & 0.2021 & 0.0610 & 0.5283 & $0.040^{*}$ \\
\hline $\mathrm{H} 15 \mathrm{C}$ & 0.2175 & 0.1501 & 0.5631 & $0.040^{*}$ \\
\hline C16 & $0.29273(16)$ & $0.38648(9)$ & $0.23919(8)$ & 0.0175 \\
\hline $\mathrm{C} 17$ & 0.33360 (19) & $0.31137(10)$ & 0.20977 (8) & $0.0217(3)$ \\
\hline H17 & 0.3699 & 0.2690 & 0.2389 & $0.026^{*}$ \\
\hline $\mathrm{C} 18$ & $0.32232(19)$ & $0.29741(10)$ & $0.13921(8)$ & $0.0226(3)$ \\
\hline H18 & 0.3514 & 0.2459 & 0.1207 & $0.027 *$ \\
\hline C19 & $0.26861(17)$ & $0.35823(10)$ & $0.09458(8)$ & $0.0182(3)$ \\
\hline $\mathrm{C} 20$ & $0.23537(17)$ & $0.43490(10)$ & $0.12358(8)$ & $0.0189(3)$ \\
\hline $\mathrm{H} 20$ & 0.2037 & 0.4782 & 0.0942 & $0.023 *$ \\
\hline $\mathrm{C} 21$ & 0.24764 (17) & $0.44897(9)$ & $0.19415(8)$ & $0.0191(3)$ \\
\hline $\mathrm{H} 21$ & 0.2252 & 0.5018 & 0.2123 & $0.023 *$ \\
\hline $\mathrm{C} 22$ & $0.24427(18)$ & $0.34952(10)$ & $0.01995(8)$ & $0.0202(3)$ \\
\hline $\mathrm{H} 22$ & 0.2131 & 0.3986 & -0.0022 & $0.024 *$ \\
\hline $\mathrm{C} 23$ & $0.25803(18)$ & $0.28451(10)$ & $-0.02315(8)$ & $0.0201(3)$ \\
\hline $\mathrm{C} 24$ & $0.22173(17)$ & $0.28790(10)$ & $-0.09850(8)$ & $0.0201(3)$ \\
\hline $\mathrm{C} 25$ & $0.13268(17)$ & $0.35015(10)$ & $-0.12349(8)$ & $0.0194(3)$ \\
\hline H25 & 0.0903 & 0.3882 & -0.0922 & $0.023^{*}$ \\
\hline
\end{tabular}




\begin{tabular}{|c|c|c|c|c|c|}
\hline $\mathrm{C} 26$ & $0.10621(17)$ & $0.35628(10)$ & $-0.19439(8)$ & 0.0188 & \\
\hline $\mathrm{C} 27$ & $0.16914(17)$ & $0.30063(10)$ & $-0.24085(8)$ & 0.0189 & \\
\hline $\mathrm{C} 28$ & $0.25283(18)$ & $0.23611(10)$ & $-0.21509(8)$ & $0.0213(3)$ & \\
\hline $\mathrm{C} 29$ & $0.27962(18)$ & $0.22993(10)$ & $-0.14397(8)$ & $0.0229(3)$ & \\
\hline $\mathrm{H} 29$ & 0.3372 & 0.1863 & -0.1266 & $0.027 *$ & \\
\hline $\mathrm{C} 30$ & $-0.07334(18)$ & 0.45919 (11) & $-0.17877(8)$ & $0.0228(3)$ & \\
\hline $\mathrm{H} 30 \mathrm{~A}$ & -0.1350 & 0.4201 & -0.1541 & $0.034 *$ & \\
\hline H30B & -0.1327 & 0.4966 & -0.2064 & $0.034 *$ & \\
\hline $\mathrm{H} 30 \mathrm{C}$ & -0.0175 & 0.4910 & -0.1451 & $0.034 *$ & \\
\hline C31 & $0.26452(18)$ & $0.32355(10)$ & $-0.35293(8)$ & $0.0219(3)$ & \\
\hline $\mathrm{H} 31 \mathrm{~A}$ & 0.3352 & 0.3552 & -0.3265 & $0.033 *$ & \\
\hline H31B & 0.2362 & 0.3547 & -0.3943 & $0.033 *$ & \\
\hline $\mathrm{H} 31 \mathrm{C}$ & 0.3065 & 0.2709 & -0.3671 & $0.033^{*}$ & \\
\hline C32 & $0.3951(2)$ & $0.11810(11)$ & $-0.24020(9)$ & $0.0294(4)$ & \\
\hline $\mathrm{H} 32 \mathrm{~A}$ & 0.4758 & 0.1416 & -0.2143 & $0.044^{*}$ & \\
\hline H32B & 0.4314 & 0.0873 & -0.2802 & $0.044 *$ & \\
\hline $\mathrm{H} 32 \mathrm{C}$ & 0.3409 & 0.0810 & -0.2099 & $0.044^{*}$ & \\
\hline C33 & $0.31313(19)$ & $0.20649(10)$ & $0.00030(8)$ & $0.0219(3)$ & \\
\hline $\mathrm{O} 1 \mathrm{M}$ & $0.4544(3)$ & 0.42249 (17) & $-0.16592(16)$ & $0.0371(6)$ & 0.5 \\
\hline $\mathrm{H} 1 \mathrm{M}$ & 0.3705 & 0.4054 & -0.1596 & $0.056^{*}$ & 0.5 \\
\hline $\mathrm{C} 1 \mathrm{M}$ & $0.5516(4)$ & $0.3704(3)$ & $-0.1313(2)$ & $0.0338(8)$ & 0.5 \\
\hline H1M1 & 0.6414 & 0.4001 & -0.1230 & $0.051 *$ & 0.5 \\
\hline H1M2 & 0.5705 & 0.3218 & -0.1600 & $0.051 *$ & 0.5 \\
\hline H1M3 & 0.5104 & 0.3532 & -0.0868 & $0.051 *$ & 0.5 \\
\hline
\end{tabular}

Atomic displacement parameters $\left(\AA^{2}\right)$

\begin{tabular}{lllllll}
\hline & $U^{11}$ & $U^{22}$ & $U^{33}$ & $U^{12}$ & $U^{13}$ & $U^{23}$ \\
\hline O1 & $0.0286(6)$ & $0.0255(6)$ & $0.0187(5)$ & $-0.0042(5)$ & $-0.0075(5)$ & $0.0020(5)$ \\
O2 & $0.0323(6)$ & $0.0182(5)$ & $0.0125(5)$ & $-0.0012(5)$ & $-0.0003(5)$ & $-0.0012(4)$ \\
O3 & $0.0345(7)$ & $0.0187(5)$ & $0.0181(5)$ & $-0.0004(5)$ & $-0.0002(5)$ & $-0.0042(4)$ \\
O4 & $0.0247(6)$ & $0.0275(6)$ & $0.0153(5)$ & $0.0082(5)$ & $-0.0013(5)$ & $0.0017(5)$ \\
O5 & $0.0190(6)$ & $0.0349(6)$ & $0.0118(5)$ & $0.0016(5)$ & $-0.0008(4)$ & $0.0001(5)$ \\
O6 & $0.0331(7)$ & $0.0291(6)$ & $0.0161(5)$ & $0.0110(5)$ & $-0.0011(5)$ & $-0.0044(5)$ \\
N1 & $0.0477(10)$ & $0.0227(7)$ & $0.0228(7)$ & $0.0045(7)$ & $-0.0131(7)$ & $-0.0036(6)$ \\
C1 & $0.0219(8)$ & $0.0184(7)$ & $0.0205(8)$ & $-0.0015(6)$ & $0.0034(6)$ & $-0.0030(6)$ \\
C2 & $0.0208(8)$ & $0.0193(7)$ & $0.0293(9)$ & $0.0014(6)$ & $0.0023(7)$ & $0.0014(7)$ \\
C3 & $0.0251(8)$ & $0.0239(8)$ & $0.0258(8)$ & $0.0009(7)$ & $-0.0036(7)$ & $0.0060(7)$ \\
C4 & $0.0245(8)$ & $0.0228(8)$ & $0.0165(7)$ & $-0.0033(7)$ & $-0.0049(7)$ & $0.0017(6)$ \\
C5 & $0.0200(8)$ & $0.0223(8)$ & $0.0137(7)$ & $-0.0030(6)$ & $-0.0017(6)$ & $-0.0007(6)$ \\
C6 & $0.0217(8)$ & $0.0178(7)$ & $0.0145(7)$ & $-0.0029(6)$ & $0.0008(6)$ & $-0.0011(6)$ \\
C7 & $0.0189(7)$ & $0.0183(7)$ & $0.0137(7)$ & $0.0018(6)$ & $0.0008(6)$ & $-0.0001(6)$ \\
C8 & $0.0226(8)$ & $0.0192(7)$ & $0.0172(7)$ & $0.0022(6)$ & $-0.0032(6)$ & $-0.0011(6)$ \\
C9 & $0.0252(8)$ & $0.0208(8)$ & $0.0174(7)$ & $-0.0003(6)$ & $-0.0019(6)$ & $-0.0049(6)$ \\
C10 & $0.0232(8)$ & $0.0165(7)$ & $0.0169(7)$ & $-0.0015(6)$ & $0.0018(6)$ & $-0.0057(6)$ \\
C11 & $0.0179(7)$ & $0.0192(7)$ & $0.0154(7)$ & $0.0020(6)$ & $0.0005(6)$ & $-0.0017(6)$ \\
C12 & $0.0209(8)$ & $0.0205(8)$ & $0.0158(7)$ & $0.0009(6)$ & $0.0022(6)$ & $0.0000(6)$ \\
C13 & $0.0206(7)$ & $0.0175(7)$ & $0.0183(7)$ & $0.0008(6)$ & $-0.0012(6)$ & $-0.0012(6)$
\end{tabular}


supporting information

\begin{tabular}{lllllll} 
C14 & $0.0209(8)$ & $0.0256(8)$ & $0.0295(9)$ & $0.0005(7)$ & $0.0010(7)$ & $0.0009(7)$ \\
C15 & $0.0329(9)$ & $0.0265(8)$ & $0.0201(8)$ & $-0.0039(7)$ & $0.0032(7)$ & $0.0039(7)$ \\
C16 & $0.0160(7)$ & $0.0200(7)$ & $0.0165(7)$ & $-0.0013(6)$ & $-0.0008(6)$ & $0.0002(6)$ \\
C17 & $0.0293(9)$ & $0.0201(7)$ & $0.0158(7)$ & $0.0052(7)$ & $-0.0011(6)$ & $0.0029(6)$ \\
C18 & $0.0316(9)$ & $0.0192(8)$ & $0.0172(7)$ & $0.0065(7)$ & $-0.0005(7)$ & $-0.0003(6)$ \\
C19 & $0.0194(7)$ & $0.0205(7)$ & $0.0147(7)$ & $0.0005(6)$ & $0.0011(6)$ & $0.0015(6)$ \\
C20 & $0.0196(7)$ & $0.0192(7)$ & $0.0178(7)$ & $0.0018(6)$ & $-0.0006(6)$ & $0.0040(6)$ \\
C21 & $0.0226(8)$ & $0.0164(7)$ & $0.0183(7)$ & $0.0002(6)$ & $0.0002(6)$ & $-0.0005(6)$ \\
C22 & $0.0245(8)$ & $0.0202(7)$ & $0.0159(7)$ & $0.0027(7)$ & $-0.0007(6)$ & $0.0042(6)$ \\
C23 & $0.0226(8)$ & $0.0219(8)$ & $0.0158(7)$ & $0.0015(7)$ & $-0.0013(6)$ & $0.0023(6)$ \\
C24 & $0.0237(8)$ & $0.0215(7)$ & $0.0151(7)$ & $-0.0016(6)$ & $-0.0008(6)$ & $0.0014(6)$ \\
C25 & $0.0215(8)$ & $0.0227(7)$ & $0.0140(7)$ & $0.0003(6)$ & $0.0002(6)$ & $-0.0006(6)$ \\
C26 & $0.0177(7)$ & $0.0217(7)$ & $0.0170(7)$ & $-0.0006(6)$ & $-0.0011(6)$ & $0.0024(6)$ \\
C27 & $0.0174(7)$ & $0.0263(8)$ & $0.0130(7)$ & $-0.0016(6)$ & $-0.0006(6)$ & $0.0010(6)$ \\
C28 & $0.0225(8)$ & $0.0245(8)$ & $0.0168(7)$ & $0.0003(7)$ & $0.0003(6)$ & $-0.0029(6)$ \\
C29 & $0.0277(8)$ & $0.0229(8)$ & $0.0181(8)$ & $0.0047(7)$ & $-0.0028(6)$ & $0.0007(6)$ \\
C30 & $0.0222(8)$ & $0.0269(8)$ & $0.0192(8)$ & $0.0048(7)$ & $-0.0010(6)$ & $-0.0031(7)$ \\
C31 & $0.0230(8)$ & $0.0264(8)$ & $0.0165(7)$ & $0.0005(7)$ & $0.0029(6)$ & $0.0007(6)$ \\
C32 & $0.0359(10)$ & $0.0292(9)$ & $0.0231(8)$ & $0.0125(8)$ & $-0.0005(8)$ & $-0.0024(7)$ \\
C33 & $0.0301(8)$ & $0.0225(8)$ & $0.0130(7)$ & $-0.0001(7)$ & $-0.0046(6)$ & $-0.0031(6)$ \\
O1M & $0.0289(13)$ & $0.0360(14)$ & $0.0465(16)$ & $-0.0027(12)$ & $0.0055(12)$ & $0.0064(13)$ \\
C1M & $0.0213(17)$ & $0.044(2)$ & $0.0359(19)$ & $-0.0039(15)$ & $0.0058(16)$ & $-0.0010(18)$ \\
& & & & & & \\
\hline
\end{tabular}

Geometric parameters $\left(\AA,{ }^{\circ}\right)$

\begin{tabular}{llll}
\hline $\mathrm{O} 1-\mathrm{C} 5$ & $1.4426(18)$ & $\mathrm{C} 14-\mathrm{H} 14 \mathrm{~B}$ & 0.9800 \\
$\mathrm{O} 1-\mathrm{C} 4$ & $1.454(2)$ & $\mathrm{C} 14-\mathrm{H} 14 \mathrm{C}$ & 0.9800 \\
$\mathrm{O} 2-\mathrm{C} 12$ & $1.3500(19)$ & $\mathrm{C} 15-\mathrm{H} 15 \mathrm{~A}$ & 0.9800 \\
$\mathrm{O} 2-\mathrm{C} 6$ & $1.4587(18)$ & $\mathrm{C} 15-\mathrm{H} 15 \mathrm{~B}$ & 0.9800 \\
$\mathrm{O} 3-\mathrm{C} 12$ & $1.208(2)$ & $\mathrm{C} 15-\mathrm{H} 15 \mathrm{C}$ & 0.9800 \\
$\mathrm{O} 4-\mathrm{C} 26$ & $1.3602(19)$ & $\mathrm{C} 16-\mathrm{C} 17$ & $1.398(2)$ \\
$\mathrm{O} 4-\mathrm{C} 30$ & $1.4321(19)$ & $\mathrm{C} 16-\mathrm{C} 21$ & $1.400(2)$ \\
$\mathrm{O} 5-\mathrm{C} 27$ & $1.3713(18)$ & $\mathrm{C} 17-\mathrm{C} 18$ & $1.382(2)$ \\
$\mathrm{O} 5-\mathrm{C} 31$ & $1.4381(19)$ & $\mathrm{C} 17-\mathrm{H} 17$ & 0.9500 \\
$\mathrm{O} 6-\mathrm{C} 28$ & $1.3619(19)$ & $\mathrm{C} 18-\mathrm{C} 19$ & $1.402(2)$ \\
$\mathrm{O} 6-\mathrm{C} 32$ & $1.425(2)$ & $\mathrm{C} 18-\mathrm{H} 18$ & 0.9500 \\
$\mathrm{~N} 1-\mathrm{C} 33$ & $1.151(2)$ & $\mathrm{C} 19-\mathrm{C} 20$ & $1.400(2)$ \\
$\mathrm{C} 1-\mathrm{C} 10$ & $1.334(2)$ & $\mathrm{C} 19-\mathrm{C} 22$ & $1.462(2)$ \\
$\mathrm{C} 1-\mathrm{C} 2$ & $1.508(2)$ & $\mathrm{C} 20-\mathrm{C} 21$ & $1.383(2)$ \\
$\mathrm{C} 1-\mathrm{H} 1$ & 0.9500 & $\mathrm{C} 20-\mathrm{H} 20$ & 0.9500 \\
$\mathrm{C} 2-\mathrm{C} 3$ & $1.547(2)$ & $\mathrm{C} 21-\mathrm{H} 21$ & 0.9500 \\
$\mathrm{C} 2-\mathrm{H} 2 \mathrm{~A}$ & 0.9900 & $\mathrm{C} 22-\mathrm{C} 23$ & $1.349(2)$ \\
$\mathrm{C} 2-\mathrm{H} 2 \mathrm{~B}$ & 0.9900 & $\mathrm{C} 22-\mathrm{H} 22$ & 0.9500 \\
$\mathrm{C} 3-\mathrm{C} 4$ & $1.507(2)$ & $\mathrm{C} 23-\mathrm{C} 33$ & $1.440(2)$ \\
$\mathrm{C} 3-\mathrm{H} 3 \mathrm{~A}$ & 0.9900 & $\mathrm{C} 23-\mathrm{C} 24$ & $1.491(2)$ \\
$\mathrm{C} 3-\mathrm{H} 3 \mathrm{~B}$ & 0.9900 & $\mathrm{C} 24-\mathrm{C} 29$ & $1.395(2)$ \\
$\mathrm{C} 4-\mathrm{C} 5$ & $1.470(2)$ & $\mathrm{C} 24-\mathrm{C} 25$ & $1.395(2)$ \\
$\mathrm{C} 4-\mathrm{C} 15$ & $1.505(2)$ & $\mathrm{C} 25-\mathrm{C} 26$ & $1.391(2)$ \\
& & &
\end{tabular}




\begin{tabular}{|c|c|c|c|}
\hline $\mathrm{C} 5-\mathrm{C} 6$ & $1.506(2)$ & $\mathrm{C} 25-\mathrm{H} 25$ & 0.9500 \\
\hline $\mathrm{C} 5-\mathrm{H} 5$ & 1.0000 & $\mathrm{C} 26-\mathrm{C} 27$ & $1.401(2)$ \\
\hline $\mathrm{C} 6-\mathrm{C} 7$ & $1.558(2)$ & $\mathrm{C} 27-\mathrm{C} 28$ & $1.398(2)$ \\
\hline $\mathrm{C} 6-\mathrm{H} 6$ & 1.0000 & $\mathrm{C} 28-\mathrm{C} 29$ & $1.396(2)$ \\
\hline $\mathrm{C} 7-\mathrm{C} 11$ & $1.511(2)$ & $\mathrm{C} 29-\mathrm{H} 29$ & 0.9500 \\
\hline $\mathrm{C} 7-\mathrm{C} 8$ & $1.544(2)$ & $\mathrm{C} 30-\mathrm{H} 30 \mathrm{~A}$ & 0.9800 \\
\hline $\mathrm{C} 7-\mathrm{H} 7$ & 1.0000 & $\mathrm{C} 30-\mathrm{H} 30 \mathrm{~B}$ & 0.9800 \\
\hline $\mathrm{C} 8-\mathrm{C} 9$ & $1.540(2)$ & $\mathrm{C} 30-\mathrm{H} 30 \mathrm{C}$ & 0.9800 \\
\hline $\mathrm{C} 8-\mathrm{H} 8 \mathrm{~A}$ & 0.9900 & $\mathrm{C} 31-\mathrm{H} 31 \mathrm{~A}$ & 0.9800 \\
\hline C $8-\mathrm{H} 8 \mathrm{~B}$ & 0.9900 & $\mathrm{C} 31-\mathrm{H} 31 \mathrm{~B}$ & 0.9800 \\
\hline C9-C10 & $1.508(2)$ & $\mathrm{C} 31-\mathrm{H} 31 \mathrm{C}$ & 0.9800 \\
\hline C9-H9A & 0.9900 & $\mathrm{C} 32-\mathrm{H} 32 \mathrm{~A}$ & 0.9800 \\
\hline C9-H9B & 0.9900 & $\mathrm{C} 32-\mathrm{H} 32 \mathrm{~B}$ & 0.9800 \\
\hline $\mathrm{C} 10-\mathrm{C} 14$ & $1.505(2)$ & $\mathrm{C} 32-\mathrm{H} 32 \mathrm{C}$ & 0.9800 \\
\hline $\mathrm{C} 11-\mathrm{C} 13$ & $1.338(2)$ & $\mathrm{O} 1 \mathrm{M}-\mathrm{C} 1 \mathrm{M}$ & $1.408(5)$ \\
\hline $\mathrm{C} 11-\mathrm{C} 12$ & $1.484(2)$ & $\mathrm{O} 1 \mathrm{M}-\mathrm{H} 1 \mathrm{M}$ & 0.8400 \\
\hline $\mathrm{C} 13-\mathrm{C} 16$ & $1.466(2)$ & $\mathrm{C} 1 \mathrm{M}-\mathrm{H} 1 \mathrm{M} 1$ & 0.9800 \\
\hline $\mathrm{C} 13-\mathrm{H} 13$ & 0.9500 & $\mathrm{C} 1 \mathrm{M}-\mathrm{H} 1 \mathrm{M} 2$ & 0.9800 \\
\hline C14-H14A & 0.9800 & $\mathrm{C} 1 \mathrm{M}-\mathrm{H} 1 \mathrm{M} 3$ & 0.9800 \\
\hline $\mathrm{C} 5-\mathrm{O} 1-\mathrm{C} 4$ & $60.96(10)$ & $\mathrm{C} 4-\mathrm{C} 15-\mathrm{H} 15 \mathrm{~A}$ & 109.5 \\
\hline $\mathrm{C} 12-\mathrm{O} 2-\mathrm{C} 6$ & $111.14(11)$ & $\mathrm{C} 4-\mathrm{C} 15-\mathrm{H} 15 \mathrm{~B}$ & 109.5 \\
\hline $\mathrm{C} 26-\mathrm{O} 4-\mathrm{C} 30$ & $117.40(12)$ & $\mathrm{H} 15 \mathrm{~A}-\mathrm{C} 15-\mathrm{H} 15 \mathrm{~B}$ & 109.5 \\
\hline $\mathrm{C} 27-\mathrm{O} 5-\mathrm{C} 31$ & $114.60(12)$ & $\mathrm{C} 4-\mathrm{C} 15-\mathrm{H} 15 \mathrm{C}$ & 109.5 \\
\hline $\mathrm{C} 28-\mathrm{O} 6-\mathrm{C} 32$ & $117.42(12)$ & $\mathrm{H} 15 \mathrm{~A}-\mathrm{C} 15-\mathrm{H} 15 \mathrm{C}$ & 109.5 \\
\hline $\mathrm{C} 10-\mathrm{C} 1-\mathrm{C} 2$ & $127.16(15)$ & $\mathrm{H} 15 \mathrm{~B}-\mathrm{C} 15-\mathrm{H} 15 \mathrm{C}$ & 109.5 \\
\hline $\mathrm{C} 10-\mathrm{C} 1-\mathrm{H} 1$ & 116.4 & $\mathrm{C} 17-\mathrm{C} 16-\mathrm{C} 21$ & $117.62(14)$ \\
\hline $\mathrm{C} 2-\mathrm{C} 1-\mathrm{H} 1$ & 116.4 & $\mathrm{C} 17-\mathrm{C} 16-\mathrm{C} 13$ & $123.93(14)$ \\
\hline $\mathrm{C} 1-\mathrm{C} 2-\mathrm{C} 3$ & $111.01(13)$ & $\mathrm{C} 21-\mathrm{C} 16-\mathrm{C} 13$ & $118.42(14)$ \\
\hline $\mathrm{C} 1-\mathrm{C} 2-\mathrm{H} 2 \mathrm{~A}$ & 109.4 & $\mathrm{C} 18-\mathrm{C} 17-\mathrm{C} 16$ & $121.39(15)$ \\
\hline $\mathrm{C} 3-\mathrm{C} 2-\mathrm{H} 2 \mathrm{~A}$ & 109.4 & $\mathrm{C} 18-\mathrm{C} 17-\mathrm{H} 17$ & 119.3 \\
\hline $\mathrm{C} 1-\mathrm{C} 2-\mathrm{H} 2 \mathrm{~B}$ & 109.4 & $\mathrm{C} 16-\mathrm{C} 17-\mathrm{H} 17$ & 119.3 \\
\hline $\mathrm{C} 3-\mathrm{C} 2-\mathrm{H} 2 \mathrm{~B}$ & 109.4 & $\mathrm{C} 17-\mathrm{C} 18-\mathrm{C} 19$ & $120.96(14)$ \\
\hline $\mathrm{H} 2 \mathrm{~A}-\mathrm{C} 2-\mathrm{H} 2 \mathrm{~B}$ & 108.0 & $\mathrm{C} 17-\mathrm{C} 18-\mathrm{H} 18$ & 119.5 \\
\hline $\mathrm{C} 4-\mathrm{C} 3-\mathrm{C} 2$ & $110.34(14)$ & $\mathrm{C} 19-\mathrm{C} 18-\mathrm{H} 18$ & 119.5 \\
\hline $\mathrm{C} 4-\mathrm{C} 3-\mathrm{H} 3 \mathrm{~A}$ & 109.6 & $\mathrm{C} 20-\mathrm{C} 19-\mathrm{C} 18$ & $117.49(14)$ \\
\hline $\mathrm{C} 2-\mathrm{C} 3-\mathrm{H} 3 \mathrm{~A}$ & 109.6 & $\mathrm{C} 20-\mathrm{C} 19-\mathrm{C} 22$ & $116.35(14)$ \\
\hline $\mathrm{C} 4-\mathrm{C} 3-\mathrm{H} 3 \mathrm{~B}$ & 109.6 & $\mathrm{C} 18-\mathrm{C} 19-\mathrm{C} 22$ & $126.16(15)$ \\
\hline $\mathrm{C} 2-\mathrm{C} 3-\mathrm{H} 3 \mathrm{~B}$ & 109.6 & $\mathrm{C} 21-\mathrm{C} 20-\mathrm{C} 19$ & $121.39(14)$ \\
\hline $\mathrm{H} 3 \mathrm{~A}-\mathrm{C} 3-\mathrm{H} 3 \mathrm{~B}$ & 108.1 & $\mathrm{C} 21-\mathrm{C} 20-\mathrm{H} 20$ & 119.3 \\
\hline $\mathrm{O} 1-\mathrm{C} 4-\mathrm{C} 5$ & $59.12(10)$ & $\mathrm{C} 19-\mathrm{C} 20-\mathrm{H} 20$ & 119.3 \\
\hline $\mathrm{O} 1-\mathrm{C} 4-\mathrm{C} 15$ & $112.24(13)$ & $\mathrm{C} 20-\mathrm{C} 21-\mathrm{C} 16$ & $120.93(14)$ \\
\hline $\mathrm{C} 5-\mathrm{C} 4-\mathrm{C} 15$ & $122.56(15)$ & $\mathrm{C} 20-\mathrm{C} 21-\mathrm{H} 21$ & 119.5 \\
\hline $\mathrm{O} 1-\mathrm{C} 4-\mathrm{C} 3$ & $117.45(14)$ & $\mathrm{C} 16-\mathrm{C} 21-\mathrm{H} 21$ & 119.5 \\
\hline $\mathrm{C} 5-\mathrm{C} 4-\mathrm{C} 3$ & $116.05(14)$ & $\mathrm{C} 23-\mathrm{C} 22-\mathrm{C} 19$ & $131.73(15)$ \\
\hline $\mathrm{C} 15-\mathrm{C} 4-\mathrm{C} 3$ & $116.40(14)$ & $\mathrm{C} 23-\mathrm{C} 22-\mathrm{H} 22$ & 114.1 \\
\hline $\mathrm{O} 1-\mathrm{C} 5-\mathrm{C} 4$ & $59.92(10)$ & $\mathrm{C} 19-\mathrm{C} 22-\mathrm{H} 22$ & 114.1 \\
\hline $\mathrm{O} 1-\mathrm{C} 5-\mathrm{C} 6$ & 121.09 (13) & $\mathrm{C} 22-\mathrm{C} 23-\mathrm{C} 33$ & $122.00(14)$ \\
\hline
\end{tabular}




\begin{tabular}{|c|c|c|c|}
\hline $\mathrm{C} 4-\mathrm{C} 5-\mathrm{C} 6$ & $124.78(14)$ & $\mathrm{C} 22-\mathrm{C} 23-\mathrm{C} 24$ & $123.24(15)$ \\
\hline $\mathrm{O} 1-\mathrm{C} 5-\mathrm{H} 5$ & 113.6 & $\mathrm{C} 33-\mathrm{C} 23-\mathrm{C} 24$ & $114.74(14)$ \\
\hline $\mathrm{C} 4-\mathrm{C} 5-\mathrm{H} 5$ & 113.6 & $\mathrm{C} 29-\mathrm{C} 24-\mathrm{C} 25$ & $120.25(14)$ \\
\hline $\mathrm{C} 6-\mathrm{C} 5-\mathrm{H} 5$ & 113.6 & $\mathrm{C} 29-\mathrm{C} 24-\mathrm{C} 23$ & $119.87(14)$ \\
\hline $\mathrm{O} 2-\mathrm{C} 6-\mathrm{C} 5$ & $108.87(12)$ & $\mathrm{C} 25-\mathrm{C} 24-\mathrm{C} 23$ & $119.86(14)$ \\
\hline $\mathrm{O} 2-\mathrm{C} 6-\mathrm{C} 7$ & $106.71(12)$ & $\mathrm{C} 26-\mathrm{C} 25-\mathrm{C} 24$ & $119.76(15)$ \\
\hline $\mathrm{C} 5-\mathrm{C} 6-\mathrm{C} 7$ & $112.02(12)$ & $\mathrm{C} 26-\mathrm{C} 25-\mathrm{H} 25$ & 120.1 \\
\hline $\mathrm{O} 2-\mathrm{C} 6-\mathrm{H} 6$ & 109.7 & $\mathrm{C} 24-\mathrm{C} 25-\mathrm{H} 25$ & 120.1 \\
\hline $\mathrm{C} 5-\mathrm{C} 6-\mathrm{H} 6$ & 109.7 & $\mathrm{O} 4-\mathrm{C} 26-\mathrm{C} 25$ & $124.05(14)$ \\
\hline $\mathrm{C} 7-\mathrm{C} 6-\mathrm{H} 6$ & 109.7 & $\mathrm{O} 4-\mathrm{C} 26-\mathrm{C} 27$ & $115.54(13)$ \\
\hline $\mathrm{C} 11-\mathrm{C} 7-\mathrm{C} 8$ & $114.26(13)$ & $\mathrm{C} 25-\mathrm{C} 26-\mathrm{C} 27$ & $120.41(15)$ \\
\hline $\mathrm{C} 11-\mathrm{C} 7-\mathrm{C} 6$ & $102.28(12)$ & $\mathrm{O} 5-\mathrm{C} 27-\mathrm{C} 28$ & $121.77(14)$ \\
\hline $\mathrm{C} 8-\mathrm{C} 7-\mathrm{C} 6$ & $114.66(13)$ & $\mathrm{O} 5-\mathrm{C} 27-\mathrm{C} 26$ & $118.71(14)$ \\
\hline $\mathrm{C} 11-\mathrm{C} 7-\mathrm{H} 7$ & 108.4 & $\mathrm{C} 28-\mathrm{C} 27-\mathrm{C} 26$ & $119.43(14)$ \\
\hline $\mathrm{C} 8-\mathrm{C} 7-\mathrm{H} 7$ & 108.4 & $\mathrm{O} 6-\mathrm{C} 28-\mathrm{C} 29$ & $124.43(15)$ \\
\hline $\mathrm{C} 6-\mathrm{C} 7-\mathrm{H} 7$ & 108.4 & $\mathrm{O} 6-\mathrm{C} 28-\mathrm{C} 27$ & $115.43(13)$ \\
\hline $\mathrm{C} 9-\mathrm{C} 8-\mathrm{C} 7$ & $113.36(13)$ & $\mathrm{C} 29-\mathrm{C} 28-\mathrm{C} 27$ & $120.14(14)$ \\
\hline $\mathrm{C} 9-\mathrm{C} 8-\mathrm{H} 8 \mathrm{~A}$ & 108.9 & $\mathrm{C} 24-\mathrm{C} 29-\mathrm{C} 28$ & $119.87(15)$ \\
\hline $\mathrm{C} 7-\mathrm{C} 8-\mathrm{H} 8 \mathrm{~A}$ & 108.9 & $\mathrm{C} 24-\mathrm{C} 29-\mathrm{H} 29$ & 120.1 \\
\hline $\mathrm{C} 9-\mathrm{C} 8-\mathrm{H} 8 \mathrm{~B}$ & 108.9 & $\mathrm{C} 28-\mathrm{C} 29-\mathrm{H} 29$ & 120.1 \\
\hline $\mathrm{C} 7-\mathrm{C} 8-\mathrm{H} 8 \mathrm{~B}$ & 108.9 & $\mathrm{O} 4-\mathrm{C} 30-\mathrm{H} 30 \mathrm{~A}$ & 109.5 \\
\hline $\mathrm{H} 8 \mathrm{~A}-\mathrm{C} 8-\mathrm{H} 8 \mathrm{~B}$ & 107.7 & $\mathrm{O} 4-\mathrm{C} 30-\mathrm{H} 30 \mathrm{~B}$ & 109.5 \\
\hline $\mathrm{C} 10-\mathrm{C} 9-\mathrm{C} 8$ & $113.42(12)$ & $\mathrm{H} 30 \mathrm{~A}-\mathrm{C} 30-\mathrm{H} 30 \mathrm{~B}$ & 109.5 \\
\hline $\mathrm{C} 10-\mathrm{C} 9-\mathrm{H} 9 \mathrm{~A}$ & 108.9 & $\mathrm{O} 4-\mathrm{C} 30-\mathrm{H} 30 \mathrm{C}$ & 109.5 \\
\hline $\mathrm{C} 8-\mathrm{C} 9-\mathrm{H} 9 \mathrm{~A}$ & 108.9 & $\mathrm{H} 30 \mathrm{~A}-\mathrm{C} 30-\mathrm{H} 30 \mathrm{C}$ & 109.5 \\
\hline $\mathrm{C} 10-\mathrm{C} 9-\mathrm{H} 9 \mathrm{~B}$ & 108.9 & $\mathrm{H} 30 \mathrm{~B}-\mathrm{C} 30-\mathrm{H} 30 \mathrm{C}$ & 109.5 \\
\hline $\mathrm{C} 8-\mathrm{C} 9-\mathrm{H} 9 \mathrm{~B}$ & 108.9 & $\mathrm{O} 5-\mathrm{C} 31-\mathrm{H} 31 \mathrm{~A}$ & 109.5 \\
\hline $\mathrm{H} 9 \mathrm{~A}-\mathrm{C} 9-\mathrm{H} 9 \mathrm{~B}$ & 107.7 & $\mathrm{O} 5-\mathrm{C} 31-\mathrm{H} 31 \mathrm{~B}$ & 109.5 \\
\hline $\mathrm{C} 1-\mathrm{C} 10-\mathrm{C} 14$ & $125.32(15)$ & $\mathrm{H} 31 \mathrm{~A}-\mathrm{C} 31-\mathrm{H} 31 \mathrm{~B}$ & 109.5 \\
\hline $\mathrm{C} 1-\mathrm{C} 10-\mathrm{C} 9$ & $120.92(15)$ & $\mathrm{O} 5-\mathrm{C} 31-\mathrm{H} 31 \mathrm{C}$ & 109.5 \\
\hline $\mathrm{C} 14-\mathrm{C} 10-\mathrm{C} 9$ & $113.76(14)$ & $\mathrm{H} 31 \mathrm{~A}-\mathrm{C} 31-\mathrm{H} 31 \mathrm{C}$ & 109.5 \\
\hline $\mathrm{C} 13-\mathrm{C} 11-\mathrm{C} 12$ & $118.88(14)$ & $\mathrm{H} 31 \mathrm{~B}-\mathrm{C} 31-\mathrm{H} 31 \mathrm{C}$ & 109.5 \\
\hline $\mathrm{C} 13-\mathrm{C} 11-\mathrm{C} 7$ & $132.60(14)$ & $\mathrm{O} 6-\mathrm{C} 32-\mathrm{H} 32 \mathrm{~A}$ & 109.5 \\
\hline $\mathrm{C} 12-\mathrm{C} 11-\mathrm{C} 7$ & $108.40(13)$ & $\mathrm{O} 6-\mathrm{C} 32-\mathrm{H} 32 \mathrm{~B}$ & 109.5 \\
\hline $\mathrm{O} 3-\mathrm{C} 12-\mathrm{O} 2$ & $121.34(14)$ & $\mathrm{H} 32 \mathrm{~A}-\mathrm{C} 32-\mathrm{H} 32 \mathrm{~B}$ & 109.5 \\
\hline $\mathrm{O} 3-\mathrm{C} 12-\mathrm{C} 11$ & $128.93(15)$ & $\mathrm{O} 6-\mathrm{C} 32-\mathrm{H} 32 \mathrm{C}$ & 109.5 \\
\hline $\mathrm{O} 2-\mathrm{C} 12-\mathrm{C} 11$ & $109.63(13)$ & $\mathrm{H} 32 \mathrm{~A}-\mathrm{C} 32-\mathrm{H} 32 \mathrm{C}$ & 109.5 \\
\hline $\mathrm{C} 11-\mathrm{C} 13-\mathrm{C} 16$ & $130.38(15)$ & $\mathrm{H} 32 \mathrm{~B}-\mathrm{C} 32-\mathrm{H} 32 \mathrm{C}$ & 109.5 \\
\hline $\mathrm{C} 11-\mathrm{C} 13-\mathrm{H} 13$ & 114.8 & $\mathrm{~N} 1-\mathrm{C} 33-\mathrm{C} 23$ & $177.04(16)$ \\
\hline $\mathrm{C} 16-\mathrm{C} 13-\mathrm{H} 13$ & 114.8 & $\mathrm{C} 1 \mathrm{M}-\mathrm{O} 1 \mathrm{M}-\mathrm{H} 1 \mathrm{M}$ & 109.5 \\
\hline $\mathrm{C} 10-\mathrm{C} 14-\mathrm{H} 14 \mathrm{~A}$ & 109.5 & $\mathrm{O} 1 \mathrm{M}-\mathrm{C} 1 \mathrm{M}-\mathrm{H} 1 \mathrm{M} 1$ & 109.5 \\
\hline $\mathrm{C} 10-\mathrm{C} 14-\mathrm{H} 14 \mathrm{~B}$ & 109.5 & $\mathrm{O} 1 \mathrm{M}-\mathrm{C} 1 \mathrm{M}-\mathrm{H} 1 \mathrm{M} 2$ & 109.5 \\
\hline $\mathrm{H} 14 \mathrm{~A}-\mathrm{C} 14-\mathrm{H} 14 \mathrm{~B}$ & 109.5 & $\mathrm{H} 1 \mathrm{M} 1-\mathrm{C} 1 \mathrm{M}-\mathrm{H} 1 \mathrm{M} 2$ & 109.5 \\
\hline $\mathrm{C} 10-\mathrm{C} 14-\mathrm{H} 14 \mathrm{C}$ & 109.5 & $\mathrm{O} 1 \mathrm{M}-\mathrm{C} 1 \mathrm{M}-\mathrm{H} 1 \mathrm{M} 3$ & 109.5 \\
\hline $\mathrm{H} 14 \mathrm{~A}-\mathrm{C} 14-\mathrm{H} 14 \mathrm{C}$ & 109.5 & $\mathrm{H} 1 \mathrm{M} 1-\mathrm{C} 1 \mathrm{M}-\mathrm{H} 1 \mathrm{M} 3$ & 109.5 \\
\hline $\mathrm{H} 14 \mathrm{~B}-\mathrm{C} 14-\mathrm{H} 14 \mathrm{C}$ & 109.5 & $\mathrm{H} 1 \mathrm{M} 2-\mathrm{C} 1 \mathrm{M}-\mathrm{H} 1 \mathrm{M} 3$ & 109.5 \\
\hline $\mathrm{C} 10-\mathrm{C} 1-\mathrm{C} 2-\mathrm{C} 3$ & $-107.33(19)$ & $\mathrm{C} 11-\mathrm{C} 13-\mathrm{C} 16-\mathrm{C} 17$ & $-18.2(3)$ \\
\hline
\end{tabular}




\begin{tabular}{|c|c|c|c|}
\hline $\mathrm{C} 1-\mathrm{C} 2-\mathrm{C} 3-\mathrm{C} 4$ & $51.42(18)$ & $\mathrm{C} 11-\mathrm{C} 13-\mathrm{C} 16-\mathrm{C} 21$ & $163.60(17)$ \\
\hline $\mathrm{C} 5-\mathrm{O} 1-\mathrm{C} 4-\mathrm{C} 15$ & $-115.66(16)$ & $\mathrm{C} 21-\mathrm{C} 16-\mathrm{C} 17-\mathrm{C} 18$ & $-3.6(2)$ \\
\hline $\mathrm{C} 5-\mathrm{O} 1-\mathrm{C} 4-\mathrm{C} 3$ & $105.42(16)$ & $\mathrm{C} 13-\mathrm{C} 16-\mathrm{C} 17-\mathrm{C} 18$ & $178.17(16)$ \\
\hline $\mathrm{C} 2-\mathrm{C} 3-\mathrm{C} 4-\mathrm{O} 1$ & $-154.06(13)$ & $\mathrm{C} 16-\mathrm{C} 17-\mathrm{C} 18-\mathrm{C} 19$ & $-0.3(3)$ \\
\hline $\mathrm{C} 2-\mathrm{C} 3-\mathrm{C} 4-\mathrm{C} 5$ & $-87.00(17)$ & $\mathrm{C} 17-\mathrm{C} 18-\mathrm{C} 19-\mathrm{C} 20$ & $3.8(2)$ \\
\hline $\mathrm{C} 2-\mathrm{C} 3-\mathrm{C} 4-\mathrm{C} 15$ & $68.71(18)$ & $\mathrm{C} 17-\mathrm{C} 18-\mathrm{C} 19-\mathrm{C} 22$ & $-176.49(17)$ \\
\hline $\mathrm{C} 4-\mathrm{O} 1-\mathrm{C} 5-\mathrm{C} 6$ & $114.86(17)$ & $\mathrm{C} 18-\mathrm{C} 19-\mathrm{C} 20-\mathrm{C} 21$ & $-3.4(2)$ \\
\hline $\mathrm{C} 15-\mathrm{C} 4-\mathrm{C} 5-\mathrm{O} 1$ & $98.13(16)$ & $\mathrm{C} 22-\mathrm{C} 19-\mathrm{C} 20-\mathrm{C} 21$ & $176.89(15)$ \\
\hline $\mathrm{C} 3-\mathrm{C} 4-\mathrm{C} 5-\mathrm{O} 1$ & $-107.79(15)$ & $\mathrm{C} 19-\mathrm{C} 20-\mathrm{C} 21-\mathrm{C} 16$ & $-0.6(2)$ \\
\hline $\mathrm{O} 1-\mathrm{C} 4-\mathrm{C} 5-\mathrm{C} 6$ & $-108.93(16)$ & $\mathrm{C} 17-\mathrm{C} 16-\mathrm{C} 21-\mathrm{C} 20$ & $4.1(2)$ \\
\hline $\mathrm{C} 15-\mathrm{C} 4-\mathrm{C} 5-\mathrm{C} 6$ & $-10.8(2)$ & $\mathrm{C} 13-\mathrm{C} 16-\mathrm{C} 21-\mathrm{C} 20$ & $-177.64(15)$ \\
\hline $\mathrm{C} 3-\mathrm{C} 4-\mathrm{C} 5-\mathrm{C} 6$ & $143.28(15)$ & $\mathrm{C} 20-\mathrm{C} 19-\mathrm{C} 22-\mathrm{C} 23$ & $-176.47(17)$ \\
\hline $\mathrm{C} 12-\mathrm{O} 2-\mathrm{C} 6-\mathrm{C} 5$ & $135.46(13)$ & $\mathrm{C} 18-\mathrm{C} 19-\mathrm{C} 22-\mathrm{C} 23$ & $3.8(3)$ \\
\hline $\mathrm{C} 12-\mathrm{O} 2-\mathrm{C} 6-\mathrm{C} 7$ & $14.37(17)$ & $\mathrm{C} 19-\mathrm{C} 22-\mathrm{C} 23-\mathrm{C} 33$ & $-4.2(3)$ \\
\hline $\mathrm{O} 1-\mathrm{C} 5-\mathrm{C} 6-\mathrm{O} 2$ & $44.08(18)$ & $\mathrm{C} 19-\mathrm{C} 22-\mathrm{C} 23-\mathrm{C} 24$ & $177.52(16)$ \\
\hline $\mathrm{C} 4-\mathrm{C} 5-\mathrm{C} 6-\mathrm{O} 2$ & $116.99(15)$ & $\mathrm{C} 22-\mathrm{C} 23-\mathrm{C} 24-\mathrm{C} 29$ & $159.30(17)$ \\
\hline $\mathrm{O} 1-\mathrm{C} 5-\mathrm{C} 6-\mathrm{C} 7$ & $161.85(13)$ & $\mathrm{C} 33-\mathrm{C} 23-\mathrm{C} 24-\mathrm{C} 29$ & $-19.1(2)$ \\
\hline $\mathrm{C} 4-\mathrm{C} 5-\mathrm{C} 6-\mathrm{C} 7$ & $-125.24(16)$ & $\mathrm{C} 22-\mathrm{C} 23-\mathrm{C} 24-\mathrm{C} 25$ & $-19.2(3)$ \\
\hline $\mathrm{O} 2-\mathrm{C} 6-\mathrm{C} 7-\mathrm{C} 11$ & $-11.56(16)$ & $\mathrm{C} 33-\mathrm{C} 23-\mathrm{C} 24-\mathrm{C} 25$ & $162.36(15)$ \\
\hline $\mathrm{C} 5-\mathrm{C} 6-\mathrm{C} 7-\mathrm{C} 11$ & $-130.62(13)$ & $\mathrm{C} 29-\mathrm{C} 24-\mathrm{C} 25-\mathrm{C} 26$ & $-2.5(2)$ \\
\hline $\mathrm{O} 2-\mathrm{C} 6-\mathrm{C} 7-\mathrm{C} 8$ & $-135.79(13)$ & $\mathrm{C} 23-\mathrm{C} 24-\mathrm{C} 25-\mathrm{C} 26$ & $175.96(15)$ \\
\hline $\mathrm{C} 5-\mathrm{C} 6-\mathrm{C} 7-\mathrm{C} 8$ & $105.15(15)$ & $\mathrm{C} 30-\mathrm{O} 4-\mathrm{C} 26-\mathrm{C} 25$ & $-16.8(2)$ \\
\hline $\mathrm{C} 11-\mathrm{C} 7-\mathrm{C} 8-\mathrm{C} 9$ & $147.81(13)$ & $\mathrm{C} 30-\mathrm{O} 4-\mathrm{C} 26-\mathrm{C} 27$ & $164.02(14)$ \\
\hline $\mathrm{C} 6-\mathrm{C} 7-\mathrm{C} 8-\mathrm{C} 9$ & $-94.58(15)$ & $\mathrm{C} 24-\mathrm{C} 25-\mathrm{C} 26-\mathrm{O} 4$ & $-179.51(15)$ \\
\hline $\mathrm{C} 7-\mathrm{C} 8-\mathrm{C} 9-\mathrm{C} 10$ & $70.67(17)$ & $\mathrm{C} 24-\mathrm{C} 25-\mathrm{C} 26-\mathrm{C} 27$ & $-0.4(2)$ \\
\hline $\mathrm{C} 2-\mathrm{C} 1-\mathrm{C} 10-\mathrm{C} 14$ & $-8.4(3)$ & $\mathrm{C} 31-\mathrm{O} 5-\mathrm{C} 27-\mathrm{C} 28$ & $-63.9(2)$ \\
\hline $\mathrm{C} 2-\mathrm{C} 1-\mathrm{C} 10-\mathrm{C} 9$ & $171.04(14)$ & $\mathrm{C} 31-\mathrm{O} 5-\mathrm{C} 27-\mathrm{C} 26$ & $119.53(16)$ \\
\hline $\mathrm{C} 8-\mathrm{C} 9-\mathrm{C} 10-\mathrm{C} 1$ & $-104.35(17)$ & $\mathrm{O} 4-\mathrm{C} 26-\mathrm{C} 27-\mathrm{O} 5$ & $-0.7(2)$ \\
\hline $\mathrm{C} 8-\mathrm{C} 9-\mathrm{C} 10-\mathrm{C} 14$ & $75.17(18)$ & $\mathrm{C} 25-\mathrm{C} 26-\mathrm{C} 27-\mathrm{O} 5$ & $-179.93(14)$ \\
\hline $\mathrm{C} 8-\mathrm{C} 7-\mathrm{C} 11-\mathrm{C} 13$ & $-54.0(2)$ & $\mathrm{O} 4-\mathrm{C} 26-\mathrm{C} 27-\mathrm{C} 28$ & $-177.44(14)$ \\
\hline $\mathrm{C} 6-\mathrm{C} 7-\mathrm{C} 11-\mathrm{C} 13$ & $-178.52(17)$ & $\mathrm{C} 25-\mathrm{C} 26-\mathrm{C} 27-\mathrm{C} 28$ & $3.4(2)$ \\
\hline $\mathrm{C} 8-\mathrm{C} 7-\mathrm{C} 11-\mathrm{C} 12$ & $130.10(14)$ & $\mathrm{C} 32-\mathrm{O} 6-\mathrm{C} 28-\mathrm{C} 29$ & $-2.5(2)$ \\
\hline $\mathrm{C} 6-\mathrm{C} 7-\mathrm{C} 11-\mathrm{C} 12$ & $5.60(16)$ & $\mathrm{C} 32-\mathrm{O} 6-\mathrm{C} 28-\mathrm{C} 27$ & $177.21(15)$ \\
\hline $\mathrm{C} 6-\mathrm{O} 2-\mathrm{C} 12-\mathrm{O} 3$ & $172.42(15)$ & $\mathrm{O} 5-\mathrm{C} 27-\mathrm{C} 28-\mathrm{O} 6$ & $0.3(2)$ \\
\hline $\mathrm{C} 6-\mathrm{O} 2-\mathrm{C} 12-\mathrm{C} 11$ & $-10.86(17)$ & $\mathrm{C} 26-\mathrm{C} 27-\mathrm{C} 28-\mathrm{O} 6$ & $176.89(14)$ \\
\hline $\mathrm{C} 13-\mathrm{C} 11-\mathrm{C} 12-\mathrm{O} 3$ & $2.6(3)$ & $\mathrm{O} 5-\mathrm{C} 27-\mathrm{C} 28-\mathrm{C} 29$ & $179.97(15)$ \\
\hline $\mathrm{C} 7-\mathrm{C} 11-\mathrm{C} 12-\mathrm{O} 3$ & $179.18(17)$ & $\mathrm{C} 26-\mathrm{C} 27-\mathrm{C} 28-\mathrm{C} 29$ & $-3.4(2)$ \\
\hline $\mathrm{C} 13-\mathrm{C} 11-\mathrm{C} 12-\mathrm{O} 2$ & $-173.76(14)$ & $\mathrm{C} 25-\mathrm{C} 24-\mathrm{C} 29-\mathrm{C} 28$ & $2.5(2)$ \\
\hline $\mathrm{C} 7-\mathrm{C} 11-\mathrm{C} 12-\mathrm{O} 2$ & $2.78(18)$ & $\mathrm{C} 23-\mathrm{C} 24-\mathrm{C} 29-\mathrm{C} 28$ & $-176.03(15)$ \\
\hline $\mathrm{C} 12-\mathrm{C} 11-\mathrm{C} 13-\mathrm{C} 16$ & $170.76(16)$ & $\mathrm{O} 6-\mathrm{C} 28-\mathrm{C} 29-\mathrm{C} 24$ & $-179.82(16)$ \\
\hline $\mathrm{C} 7-\mathrm{C} 11-\mathrm{C} 13-\mathrm{C} 16$ & $-4.8(3)$ & $\mathrm{C} 27-\mathrm{C} 28-\mathrm{C} 29-\mathrm{C} 24$ & $0.5(2)$ \\
\hline
\end{tabular}

